\title{
Virtual Visitation: Conceptualization and Metrics for Digital Twinning of Large Interactive Social Events
}

\author{
Kai Riemer \\ University of Sydney \\ kai.riemer@sydney.edu.au
}

\author{
Mike Seymour \\ University of Sydney \\ mike.seymour@sydney.edu.au
}

\begin{abstract}
The COVID-19 pandemic has accelerated a move towards digital engagement in many parts of life. At the same time, it has halted large public gatherings such as music, sports or arts events. In this paper we discuss opportunities for such large- scale social events to create digital experiences that mirror, mimic or enhance traditional experiences, with new forms of digital twinning. Originating from the world of manufacturing, and popularized by the Industry 4.0 initiative, digital twins refer to the creation of digital representations of physical entities. In the context of large social events digital twins denote digital spaces inhabitable by visitors with their own digital twins in the form of avatars. In this paper we investigate how event coordinators can account for different kinds of social engagements in digital twinning spaces. We develop a framework for user interactions along two dimensions: interactions with the space itself, and social interactions between virtual visitors (avatars). Accounting for virtual visitations is more challenging but offers unique opportunities compared to traditional attendance. We present four metrics, Views, Visits, Active Visits, and Interactive Visits, and we discuss considerations for implementing advanced digital social events.
\end{abstract}

\section{Introduction}

The COVID-19 pandemic has put a halt on large public gatherings, such as concerts, exhibitions or sporting events, and closed down cultural facilities such as museums or performance spaces. As the same time, the move to remote work has spurred an unprecedented growth in the use of digital tools for communication, collaboration and engagement.

As more and more engagement in large open and interactive events in sports and culture moves online, or is supported by digital experiences, questions arise about how to account for such digital engagement. The same is true for fully digital experiences such as virtual concerts, such as recently performed inside Epic Games' Fortnite [2]. In these online concerts, millions of participants experience the concert event, and their avatars can move freely, express themselves with dance animation loops and interact with other participants inside the virtual world the game affords. The simplest analytic for the 2020 Travis Fortnite Concert was that it was 'attended' 45.8 million times, with many players known to have attended at least twice. This statistic is impressive but alone it fails to capture the richness of the experience.

Before the pandemic, large localized social events, such as live concerts, festivals, exhibitions, installations and event tourism were widely proliferating [3]. Such events frequently attracted a broad, and often global, interest beyond their immediate local context. Hence, event organizers started experimenting with new digital means to broaden their reach to capture such interest, driven by both commercial interest and social inclusion, such as to enable participation by stakeholders that would normally be unable to attend these events in person.

One digital approach to integrate physically dispersed audiences into large events is digital twinning, which is a partial or fully digital representation of a physical event, both in terms of space and activity, that is open to digital participation. These are coupled with digital only interactive events, which share many of the same attendance issues as Digital Twins.

This contrasts with fully digital worlds which in turn can also be physically extended with a real- world event, such as e-gaming tournaments, combined physical and digital location entertainment and live events with the use of AR, VR and other technology. For our purposes of exploring virtual attendance, digital twinning will be thought of as a subset of a digital-only world.

Digital twinning of social events is a new and emerging frontier for HCI, with exciting new technological possibilities, novel challenges for application and organization, as well as emerging 
research questions.

The term digital twinning has been coined in the context of digital manufacturing systems to achieve so-called Industry 4.0 standards [4][5][6]. A digital twin is thus a digital representation of a physical entity that, when coupled with the physical system, can be used to create cyber-physical systems, whereby the digital twin can be used to either simulate or control the physical one [7]. Here, we appropriate the notion of digital twinning into the social context of large events, where actual users will inhabit and interact with digital twinning spaces, effectively attending the event in digital form. What is more, not only will users interact with the digital twin space and the digital representation of the event activities but also with each other, adding an additional dimension to the digital twinning concept, not present in the original digital twins' domain.

The question arises how event organizers and organizations can create different forms of digital participation and subsequently account for such virtual visitations, via digital twins? Consequently, we ask the following research question:

How can organisations account for virtual visitation of digital events?

In order to investigate this question, we ask two further albeit related questions:

- Which digital, social interactions can be distinguished in digitally twinned or social event spaces?

- How can those forms of interaction be translated into meaning ful virtual visitation metrics?

We engage in forward looking inquiry to conceptualize the emerging phenomenon of digital twinning and large online events. We develop a framework distinguishing different kinds of user interactions in digitally twinned social event spaces along two dimensions: 1) space: different kinds of interactions with the digital space itself, and 2) social: different degrees of interactions with others in the digital space. We draw on the established notion of presence [8], the sensation of 'being there' enabled by technology, to underpin our framework.

We then illustrate our framework with the cases, including the World Expo 2021-2022 in Dubai, and discuss the practical application of the conceptual ideas in this case. We selected the World Expo as it is an event over a significant duration with a planned physical and digital presence. Unlike a sporting event that invites immediate passive viewing, the World Expo is designed for audience participation over several months. Having a clear start and finish, it is a strong candidate to study and research. Given the demands of the current global pandemic, the focus will be on the engagement of both a physical and digital audience.

We find that technological progress in interactive technology allows for new forms of previously impossible visitations to large social events via the digital space. We further find that, while challenging initially, the digital space offers more granular and sophisticated ways of accounting for different forms of virtual visitations and levels of user engagement than has been possible traditionally in the physical space. Finally, we highlight that the implementation of digital twinning and large social events, while offering new opportunities for digital inclusiveness of non-privileged audiences, depends on the technological context of intended user groups.

Our research contributes a conceptual basis for understanding user participation and measuring of virtual visitation in this emerging digital phenomenon, in the form of a framework and suggested metrics. The research is significant because it surfaces new opportunities and challenges, especially in digital participation of large off-line events via digital twin spaces. We argue that this new and emerging research frontier requires a new research agenda and contribute initial suggestions.

\section{Background}

We begin with a historical perspective on largescale social events and the motivation for considering what it means to attend such an event as a shared experience. We then introduce the idea of digital twinning to variously enhance or replicate such events in the digital world.

\subsection{Large-scale local social events}

People often travel long distances to meet at and enjoy events such as music concerts, cultural festivals, sporting events such as the Olympics, or a World Expo. Such events bring people together to share in entertainment, education, innovation, and cooperation. A long history of such events has boosted tourism and improved the image of the cities hosting them [3]. While many characteristics vary amongst these largescale events, many of them run for multiple days or weeks and require enormous effort to allow large numbers of people to participate.

People have always sought out a shared participation in major events. Such experiences are often enhanced because they are shared. Sporting and music events thrive off the excitement and presence of others who are sharing the same experience. Most would agree that an empty stadium would diminish the enjoyment of any such event, even if the game or performance was unchanged. The presence of others is 
part of what makes the event what it is, and the experience is both shared and co-created with those also in attendance.

In early 2020, COVID-19 provided unique circumstances both unexpected and unparalleled in scale. As some restrictions were lifted in countries such as Australia and New Zealand, large sporting events which would normally play to stadiums of people were played out to completely empty seat venues. With tens of thousands of completely empty seats, the sporting codes and their broadcaster added crowd audio to simulate shared involvement and even filled empty sports seats with cardboard cutouts of fans to give the illusion of a crowd. The motivation was not to deceive television audiences, but rather to address the void created by losing a sense of shared participation. Television sitcoms have done similar audio mixes of laugh tracks for decades, as had radio plays before them, to again imply mutual shared experiences, when sitcom actors were playing to empty sound stages [9].

Today, new and innovative combinations of technology promise to allow people who cannot be physically present at the event to still have a rich and meaningful engagement with the content and attractions on offer. With advances in interactive interfaces, photorealistic rendering, low-latency Internet infrastructure and advances in virtual reality (VR) we are able to create rich, meaningful interactions and shared experiences like never before. In particular, this offers new and interesting opportunities for digital engagement of audiences in large-scale shared events.

\subsection{What is digital twinning?}

A digital twin is defined as a "consistent digital representation" of "real-world objects/subjects and processes, including data transmitted by sensors" [7].

In the field of manufacturing, digital twins are at the heart of creating so-called cyber-physical systems (CPS), which integrate physical and digital components, such that a digital twin serves "as a virtual controller to the physical system," (ibid.). For example, such systems have recently been discussed in the context of digital production systems for the realization of Industry 4.0 [10]. Digital twinning is used to carry out simulations of production systems and to create representations of the production system that integrate these two parts, physical and cyber, to "a full closedloop control system, the CPS, where the physical system is controlled by the virtual one through the digital twin," [9]. Emphasis is put on the capture and representation of human activity in such production systems.

As we adapt the notion of a digital twin as a digital representation or replica of a physical event space, we extend the notion to people (subjects), but not in the sense of simulating activity, but creating digital representations of people for their interaction with the digital space. In our context digital twinning then refers to both,

1) the creation of consistent digital models of physical spaces in the form of a digital representation of a world, and

2) the digital representations of people in the form of digital avatars.

Digital twins can thus refer to both spaces and their digital representations, and people and their digital avatars.

A digital avatar is a visual presentation of an attendee or visitor. An avatar can be thought of as a digital puppet, a character that is instructed by and acts on behalf of an attendee. An avatar is defined as a, "visual representation of a human actor, which acts as a mediated stand-in or surrogate for the human actor in a virtual environment. Such visual representations can take varying levels of realism," [11].

\subsection{Digital twinning of large social events}

We envision a digital twin of a major event to be a digital replica of the event space and its main features, with the ability for people to populate this space and be present with their own personal digital twin that acts as their avatar. The exemplar we use in this paper is the World Expo 2021 to be hosted in Dubai. The Expo will feature such a large-scale digital twin of its pavilions with the ability to visit the exhibitions virtually and remotely. In the following we discuss in more general terms the possibilities we envision for such a digital twinning project.

Events such as the Expo are social events that integrate their audiences in the creation of the experience. It is thus important that the digital twin is not merely an empty space replica, but that it offers social interactivity among the patrons. The technological implementation thus imagined is not one of mere information distribution but one that creates a sense of shared presence with other people via their avatars. The technology is not an isolated end unto itself but a bridge to bring the shared experience to a much wider audience than those in Dubai.

The advances and virtual initiatives are designed to not only allow people to witness the location but to also allow others to feel "present" with them. In the following sections we will introduce the concepts of social presence and spatial presence to outline what it means to be present with others in digital twin environments.

Technically, this digital twin could be accessed in 
multiple ways on a variety of mobile, dedicated and general-purpose computer tools. Depending on bandwidth and local infrastructure this would allow a wide range of people to access, navigate and experience a real-time parallel version of the real event. Such integration allows access to specialist cameras and tools that would be embedded at the Dubai event.

We envision that local visitors would be able to access the same digital twin infrastructure to enhance their journey and gain additional insights while moving around the various parts of the physical installations. Cutting edge technology could allow users to present themselves via a customized avatar, which could interact with both curated hosts or guides as well as other attendees, physical and virtual. This could amount to an integration of the digital twin space with the physical space as well, with certain touch points acting as devices for people across physical and digital spaces to interact.

The digital twin could be envisioned as a concurrent and real-time environment with various levels of interactivity, public and private disclosure, and mediated friendship networks. Given these opportunities for creating various new forms of interactions, questions arise as to how to account for such new forms of (virtual) attendance?

New forms of engagement necessitate new forms of measurement to account for virtual visitation in its various forms. In this paper we set out to derive a framework and metrics to account for the rich and varied possibilities of virtual visitation.

\section{Framework Development}

In this section we provide a conceptual grounding to derive a metric for 'virtual attendance'. Key to defining virtual visitation will be to consider how vital the shared, social nature of the digitally enabled experience is. For example, a solo, isolated digital engagement is a poor approximation of a live attended event. With live events, people come together in a place not for convenience but for the shared experience. We thus draw on the notion of 'presence' to conceptualize different forms and degrees of 'being there' and being engaged in and with the digital event space. We begin with a discussion of different forms of presence in electronic communication.

\subsection{Presence and Awareness}

Scholars and practitioners have for some time demonstrated an interest in the concept of Information and Communications Technology (ICT)-mediated presence [12][8][13]. Generally, presence in ICT- mediated contexts is said to happen when an "illusion" occurs, such that the technology's role in the situation is suppressed from sensory awareness, resulting in a "perceptual illusion of non-mediation," [14] in which the brain is tricked into thinking it is somewhere where the body is not [14][15][8]. The idea is that technology can create an illusion in which the 'virtual' and the 'real' are confused by the mind, so that a person can be made to experience a simulated or 'virtual' situation as if they were there. For the individual, the desire is to experience a sense of spatial presence, a sense of 'being there', while being aware of their actual remote location [16][17].

Extant literature on technologically mediated presence tends to focus on how technologies and routines can be designed and implemented to create presence. For example, in a recent study on ubiquitous video conferencing arrangements at Google, the authors describe these technological configurations ("portals") as being able to "provide presence and status information on par with being co-located," [18]. Accordingly, "virtual presence" has been defined as "presence caused by virtual reality technologies," [14]. Presence in this understanding is the outcome of technological design and its use, with the assumption that the technology supports presence best when it is backgrounded; when it becomes seemingly 'invisible' to the user while supporting an illusion of attending the location or event. In other words, people connect with and enjoy the experience without being focused on the medium delivering it.

Two forms of presence can be conceptually distinguished, social presence and spatial presence, which fit the two dimensions discussed above. The first describes how people who are not physically colocated can use technology to replicate or simulate the experience of being present with each other. The second describes how people can become present in a location other than the one that they are physically located in.

With social presence, the emphasis tends to be on how technology can facilitate natural communication between two parties who are geographically distant from one another. Thus, the theory concerned with presence between people is termed social presence [19] or co-presence [20]. With spatial presence, the emphasis is on how technology can create the illusion of 'being there'. This form of presence has been defined as "a feeling of being in a location other than where you actually are" [14] and as the "suspension of disbelief that they [users of virtual reality systems] are in a world other than where their real bodies are located" [21].

Additionally, we distinguish the concept of presence awareness [22] which allows bringing the 
above two concepts together. Presence awareness is defined in the literature as, "an understanding of the activities of others, which provides a context for your own activities," [23] and helps people to align their interdependent activities [24]. Hence, presence awareness refers to the ability of an actor to perceive or be informed about the activities of other actors in a virtual space, with whom one does not necessarily have immediate interactions. It refers to the sociospatial component that goes beyond the actor's own presence with others, or their presence in a (virtual) environment. In summary we distinguish the

following three key terms:

- Social presence: the sensation that a person experiences of being present with others enabled by technology.

- Spatial presence: the sensation that technology facilitates of being present in a virtual environment.

- Presence awareness: the ability of a person to perceive the social interactions and spatial whereabouts of other actors in a virtual space, enabled by technology.

In order to use these three presence notions to conceptualize various forms of presence, we first distinguish the different actors and entities, and their relationships that are involved.

\subsection{Conceptualizing presence}

To conceptually distinguish how exactly presence is achieved in digital social events, one must consider three entities and their interactions: a focal actor, me or self (Ego); other actors (Alter); and the environment or place in question (Locum). It is assumed that all three entities are represented in the cases above.

The concepts discussed above are represented by the numbers in Figure 1, as follows:

1. My digital avatar - how the focal actor is represented and self-aware of their own representation.

2. Digital avatars of others - how other actors in the digital space are represented.

3. The digital/virtual space - how the physical space is represented.

4. Social presence- how the sensation of being present with other avatars is created.

5. Spatial presence - how the digital space is rendered present, its fidelity and the extent to which it affords manipulation.

6. Presence awareness - the extent to which the actions and interactions between others in the space are made present and accessible to the focal actor.
We assert that attainment of full socio-spatial presence will require all six aspects to be implemented in sufficient fidelity. In the next section, we will utilize the above concepts and categories for defining different degrees or forms of virtual visitation that build on the various forms of social and spatial presence.

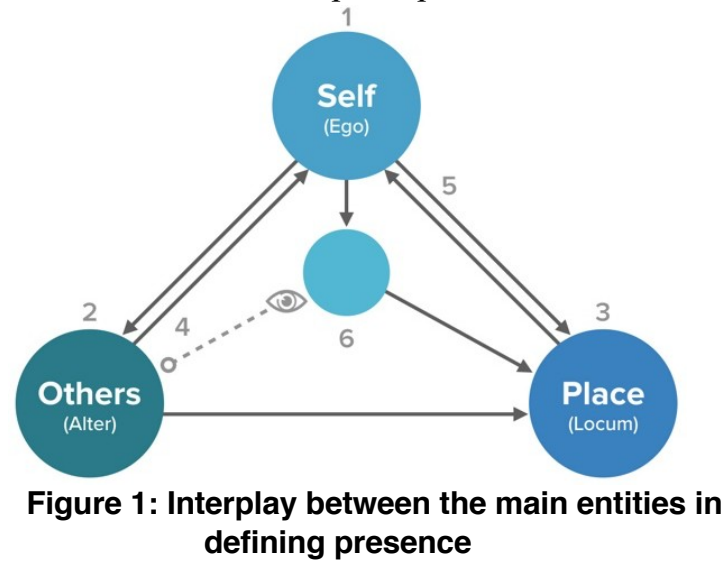

\subsection{Virtual Visitation Framework: Social and Spatial Dimensions}

Building on the concepts above, we now develop a framework for defining and distinguishing different levels of virtual visitation. In doing so, we assume first that visitation is confined initially to the digital twin space of the location in question. With increasing technical sophistication more potential for presence becomes possible, a cyber-social physical space, marks the peak of such sophistication.

The framework takes the perspective of a visitor, it models their ability to engage and experience presence, in the space. Following our conceptualization of presence (Figure 1), our framework consists of the two dimensions, social presence (presence of others - Alter) and spatial presence (presence of space - Locum), and defines different degrees of presence in each dimension, as facilitated by the platform technical capabilities.

We distinguish 3 levels of social presence:

- No presence: there is no sense of being with another; exploration is a solitary experience. You are looking at a model.

- Watch: the focal actor gets a sense of the presence of other users in the space; but this presence is mostly a passive experience of being alongside other users.

- Interact: interaction with other users is possible through various means; this provides a sense of actively being with other users in the space. Qualitatively, this sense can be richer or less so depending on the bandwidth of the technology. 


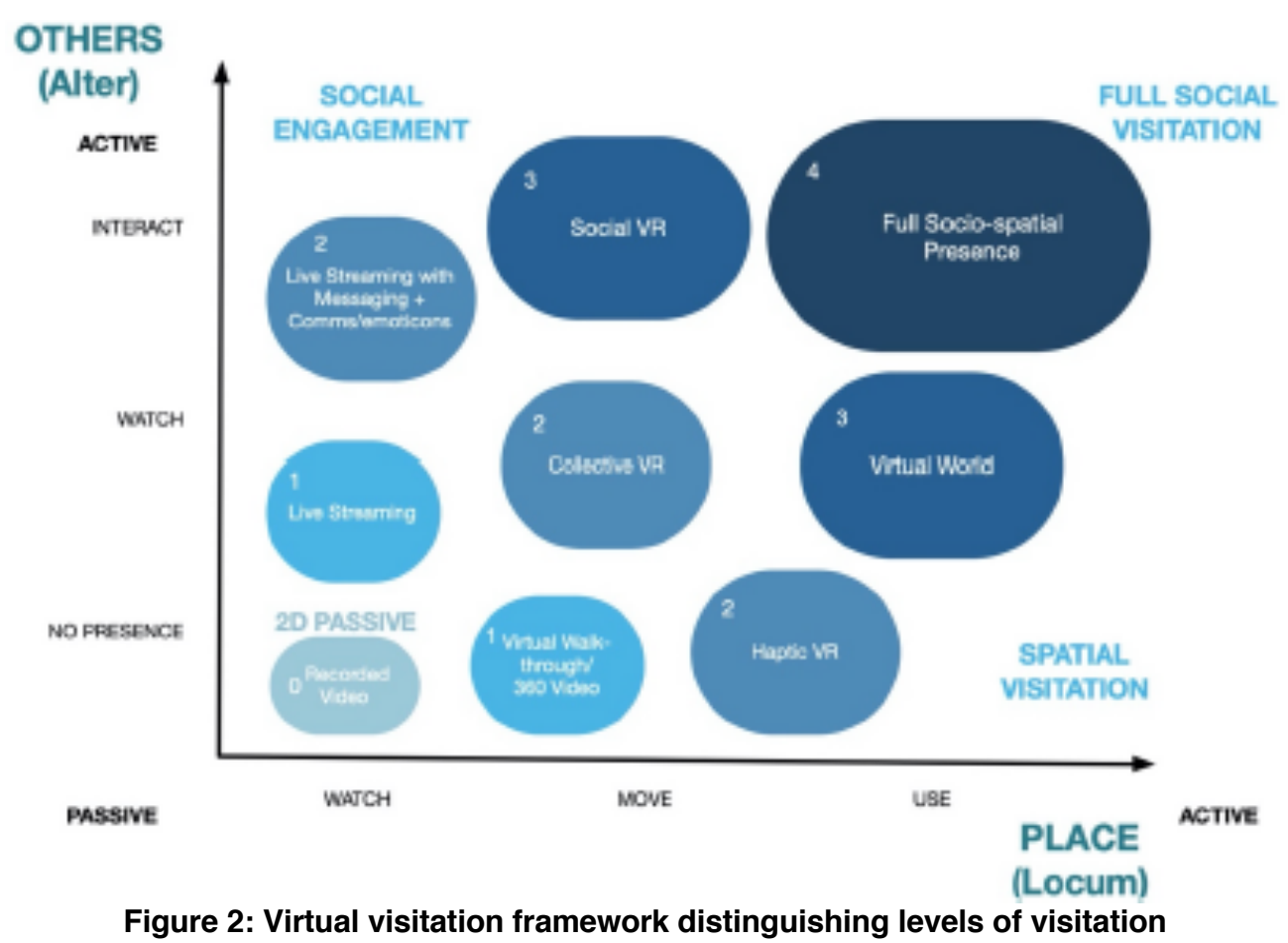

We distinguish 3 levels of spatial presence:

1. Watch: this amounts to no actual presence in the environment, merely the capability to watch as an outsider. You are watching a recording.

2. Move: the first level of actual presence comprises the ability to change position within the digital environment. This includes a sense of direction and movement of gaze and orientation. You move as a disembodied ghost, a passive observer who cannot touch.

3. Use: a higher level of presence is achieved through the ability to actively manipulate and use objects in the environment. You have a sense of being there and involvement.

\subsection{Levels of Virtual Visitation}

Levels of virtual visitation are a function of both of these two types of presence and they are often related. Concrete metrics of virtual visitation are achieved through the combination of the two dimensions, as shown in Figure 2:

Level 0 - no visitation: At this level, the user is merely presented with recorded footage, in the form of 2D video about the physical space. No noteworthy sense of presence is achieved.

Level 1 - basic visitation: This level constitutes a "visit", either in a social or spatial sense. It can be achieved in one of two ways, but both have a certain degree of passivity:

- Live-stream 2D video: Watching a live- streaming video of activity of others in the space, virtual or physical, without any ability to interact or move within the space, constitutes a basic visit. We note that the immediacy of a live video experience renders this form of engagement markedly different from pre- recorded video. People often feel they have shared an event or sporting event, if they experienced it live, even if they viewed it alone.

- Virtual walk-through: There are different ways in which a visitor might explore a digital environment, either by way of VR technology or by way of 360 degree video. While these prerecorded viewings offer degrees of freedom in terms of head movement and focus of attention, in both cases the experience is a solitary viewing with no interaction.

Level 2 - active visitation: This level of virtual visitation adds a level of activity to the experience, which leads to a sense of 'being there'. It can be achieved in three different ways:

- $\quad$ Live Messaging: The ability to interact, such as through messaging with visitors in the physical space or while watching a live stream of activity. This is akin to audience participation through social media during live television events.

- Collective VR: A basic social VR experience where the visitor is present alongside others in the digital environment. Here the visitor is able to see the activity of others live, but without the ability 
to interact.

- Haptic VR: A VR experience which affords use of features such as advanced VR implementations with haptic controls.

Level 3 - inter-active visitation: At this level of visitation, the visitor is able to interact with others, or with the environment, in meaningful ways. This is achieved in one of two ways:

- Social VR: In full social VR implementations the visitor can interact and communicate with other visitors, each represented by a digital avatar, either via text/chat or in more natural ways with natural language and/or facial expression simulation. Different degrees of fidelity are possible, whereby such fidelity might have an influence on the user experience of presence (see below).

- Virtual World: A full virtual world implementation includes the ability to manipulate the digital environment but also to observe and be affected by others' interactions with the environment (presence awareness). Yet at this level no deep or personal meaningful communication with other visitors is possible during the visitation experience. An example of this type of visitation is the user experience while playing the online game Fortnite.

Level 4 - full socio-spatial presence: This level of visitation amounts to a full social virtual world experience, whereby social interactions with others and manipulation of the environment are both possible. We label this type of visitation Full Social Visitation. We would expect that the visitor obtains full presence awareness of others' interactions with each other and the environment in a full digital twinning experience. Early examples of this kind of interaction have been signposted in some conventional social virtual worlds, such as Second Life, but at rather low-fidelity levels and not to the extent that is now possible.

\subsection{Additional factors}

The virtual visitation framework presents different levels of technically enabled visitation. The levels distinguished above can be read as features made available by the system for the user. They present potentials for visitation. The more advanced a system is, the more 'present' a visitor can potentially be in the resulting virtual space. However, potentials need not be fully utilized by any given visitor. This raises questions regarding the granularity at which visitation might be measured or accounted for in an actual systems implementation. For example, a system might offer Level 4 Visitation, yet a visitor might be content with exploring the digital space and never interacting with others. How will the system account for such a visit? How long does a visitor have to be present for a visit to count? Will there be a time threshold?

These questions are important because the digital space affords to account for visitation at a level unavailable in physical spaces. As with other evolutions of technology in areas such as media and advertising, the digital world affords greater insight into viewing and engagement. With a clearer insight comes the opportunity to better craft the user/attendee experience. We suggest that two aspects need to be considered:

First, the duration of visit, visitors to a physical location or event make the effort to travel to be there. They incur sunk cost and make a commitment which suggests they will normally spend a fair amount of time in the location. Visitors to the digital space might visit multiple times, yet only briefly each time, stringing together an experience of the event or location over several visits. This raises questions about whether these visits are separate visits or one long, yet interrupted, visit? Also, it seems sensible to establish a 'duration threshold' to weed out those short visits that cannot reasonably be counted as meaningful attendance.

Secondly, the level of engagement. In the physical world any gate entry to the park counts as a visit. Yet this crude measurement counts every entry equally, whether someone is highly interested and engaged with the event or space, or someone walks around disengaged and disinterested, such as a teenager being "dragged along" by their parents. In the digital space, activity is naturally more easily traceable, so that it is possible to account for visitation at the granular levels described above, and also taking into account the extent to which a visitor engages in either social exchanges or manipulating features of a space. Again, it appears sensible to establish a threshold to weed out those entering the space without ever moving around or engaging with the space in any way.

\subsection{Implementation options}

Additionally, different levels of visitation distinguished above deserve further consideration based on:

Fidelity of digital twinning: It stands to be expected that the fidelity and quality will have a positive effect on a visitor's experience. The framework above is agnostic to the quality with which the digital representation of physical entities, both human and non-human, is implemented. However, matters of fidelity are important. Research has shown that more realistic avatars are perceived to be more trustworthy 
and instill more affinity on social interactions, if indeed they overcome the 'uncanny valley effect', otherwise they can be decidedly negative.

Extent of the User's Technology: In conjunction with the requirements of digital representations, any implementation needs to consider the level of equipment visitors will require for a meaningful visitation. It seems advisable to allow for a range of different technologies in designing the visitor experience, such as VR glasses, mobile apps, web browsers, and with or without cameras. The user's equipment and data rates will also influence the experience. A "visit" should not be contingent on a particular technology capability to ensure inclusivity. In exactly the same way, a range of languages are supported across both the physical and digital worlds, (but similarly a minimum competence for meaningful exchanges is to be expected).

Synchronicity: A sense of events unfolding in realtime and live is a very strong influencer on one's perception of inclusion and presence. Visitors to an event such as a World Expo might join from all parts of the world and thus from different time zones. This raises questions of what happens when a visitor joins the space at a time when no physical activity is taking place to be made available digitally to the visitor (such as in the middle of the night, event location time)? A related question would be whether it was possible to 'travel in time' when attending via the digital space? For example, digital twinning enables the capturing of events to create temporal permanence, providing the ability to visit an event in a location, as if there, yet after it happened. Each individual could break the notion of 'current time'. They may be able to join a reconstruction of an interactive concert or join with others in experiencing a recreated environment which still allows interaction and participation but is timeshifted for their location. This is different from a simple recording as the event is digitally 'rebuild' to allow a new audience to explore it in their own way. Personalization: A further consideration is how the individual visitors will receive a personalized visitation experience. For example, the path or journey record that visitors will be able to save and their ability to go back to the previous visitation history to revisit their favorite places. Moreover, it is possible to receive recommendations, curated and guided tours based on personal preferences, interests, and cultural or religious background.

Privacy: While personalization of the visitation experience will require identification of the visitor, the question arises if it is possible to visit the digital environment anonymously with a pseudonym, or whether it requires giving up personal details. And how does that translate into interacting with other visitors in the digital or physical spaces? Will the visit mimic the physical space, where a visitor might have a sense of presence of a lot of strangers, but will only interact only with friends, some officials associated with the space or indeed strangers if the visitor decides to join a game, performance or otherwise give consent?

Social grouping: From the above follows that if a visitor decided to (or were mandated to) disclose their identity to the space that in turn, they should be able to share their visit with those people that they know, or at least allow them to see their presence. Besides, the space might also provide a convenient way of tracking and finding one's friends within the space. This might extend asynchronously across time (see above), in that a visitor might be allowed to retrace their friends' previous visits based on their recorded data or certain traces they define. Such social traces of visitation in the space might be saved and be accessible in the form of recommendations in certain categories to allow visitors to benefit from other people's experiences, which in turn might create yet another form of social presence.

\section{How to measure Virtual Visitation?}

To create a meaningful digital experience of any large-scale event requires careful digital twinning, and a range of decisions considering the above explicated factors. By twinning physical with digital environments, individuals will be able to have meaningful experiences in both locations, allowing a virtual attendee to exist simultaneously with a physical attendee. In doing so, comprehensive digital twinning might provide a complete and dynamic representation of the event in the digital space. As such, for those unable to physically attend, it will differ greatly from video experiences more conventionally provided. Enabling rich forms of digital attendance will necessitate new ways of accounting for such visitation, in ways that exceed the possibilities of conventional off-line counting of visitors through ticket sales or turnstile counts. Bringing to bear the conceptual framework introduced above, in the following we recommend a set of straightforward metrics intended to account for different levels of actual visitation by virtual visitors.

\subsection{Suggested Metrics}

Our recommendation is that a new system of metrics be explored that capture varying degrees of digital virtual event experiences that goes beyond traditional statistics for counting web-site usage.

In recommending a metric for virtual attendance it might seem sensible to focus on the highest level of visitation, that is Level 4 Full Social Visitation. However, this would create a situation of inequity of 
measurement. In the physical world, attendance is recorded by ticket sales or gate access. No other aspect of engagement is required to be recorded as having attended the event, other than passing through the gates. Conversely, in the digital world, there is a much lower barrier to any form of digital engagement, because in the physical world attendees must actually travel to the site, and invariably the whole exercise will cost considerably more to each individual than a virtual visit. To define full equivalence is therefore impossible. What is recommended is therefore four distinct metrics that capture attendance across levels 0 , 1,2 , and above, in our framework.

1. View: This is the traditional metric of viewing a video or web site. Additional sub metrics include the duration of view, $\%$ view to the end, and duplicate viewing. This corresponds with Visitation Level 0 in our framework and does not yet constitute a "visit". The metric is useful to capture those showing a basic interest in the event, engaging with its online materials but without entering the actual event offerings.

2. Visit: The most basic visit resides on Level 1 of our framework. While rather passive, it is reasonable to count the watching of a live stream of an event as a basic form of having attended the event. Equally, the exploration of a digital space by way of VR or 360-degree video must count as a basic notion of having visited the space. In either case we would expect a certain time threshold to apply to count as a meaningful 'visit'. We note that such forms of virtual visitation are comparable to traditional forms of physical visitation whereby a visitor is merely a bystander, walks through a space but is not necessarily engaged with the content or others in any active sense.

3. Active Visit. This is a new metric capturing Level 2 Active Visitation. As stated above, virtual visitation at this level has a certain level of activity associated with the experience, which leads to a sense of 'being there'. It can be achieved in several ways and it is an important separate metric reflecting a valuable and meaningful engagement with the environment or with others. Virtual visitors who visit together and utilize social features to share and enjoy their experience together will count as engaged.

4. Interactive Visit. This metric applies to any Level 3 Inter-active visitation or higher. Here the visitor will interact with others, or with the environment, in extensive and meaningful ways. A subset of note would be heavy engagement for Full Social Visitation. Most visitors who pass through the gates of a physical event will not engage at this level with others in this way. At the same time the digital environment offers the opportunity to capitalize on people's propensity to build social connections online and form a sense of community and even ownership that comes from contributing to the joint experience.

In conceptualizing virtual attendance, we do not propose simple equivalence with physical attendance. Even the richest digital experience will be different from a physical one. For example, there is no notion of simulating the time and effort needed to travel to a physical event, so the digital experience will always be more immediate. But there is a strong argument that delivering a digital experience is meaningful and valuable in the context of the objectives of many large public events.

Some attendees may interact more, attend for longer and be more engaged than others, based on personal preference. One great advantage with a virtual guest is our ability to better capture, track, and understand their journey and fully appreciate their level of interactivity and hence virtual presence, as reflected in the metrics above.

\section{Discussion and Outlook}

We argue that the metrics set presented above will achieve the aim of defining a metric that seeks to record meaningful virtual attendance. While retaining both a traditional metric for counting website views and a basic metric for counting visits, equivalent to counting physical attendance, it offers an additional two metrics that exploit the affordances of digital tracking in accounting for more (inter-)active forms of visitation of any virtual digital twin environment.

We note that technology is an agent of change, but not a goal in itself. Virtual attendance needs to correctly position technology in its rightful place as an enabler rather than as an end goal. The establishment of a virtual event is the skillful accomplishment that entails interactants jointly co- constructing a 'real' experience that makes them feel as if they are 'there' with other visitors.

Virtual Attendance thus recognizes that when people are present at a location, they together form the event experience. Without either shared interactions between visitors, or a rich digital environment, a meaningful virtual experience will not emerge. When these conditions are met there will be rich attendances that can be recorded and measured with the metrics introduced above. Active and interactive virtual attendances will allow capturing the significant genuine emotional exchanges that will happen, the shared laughter or a sense of awe and curiosity. The shared experiences and social presence at a location 
allows the event to 'come to life' as it turns a model or construct into an enjoyable experience worthy of being considered attending.

The most advanced forms of this virtual attendance will transcend the boundaries of both worlds and see shared emotional interaction across the virtual world into and back from the physical world. In this richest of cases, people are not only virtually attending the event but appearing in some meaningful way in the physical world, communicating with attendees physically present in at the actual event.

Hence, in the future we might envision integration of the digital twin space with the physical space at certain touch points to enable meeting and interacting between patrons on location with those visiting virtually in the digital space via their avatars.

\section{Conclusion}

The objective of this paper was to conceptualize and develop new metrics for the virtual attendance of real-world events that aim to create digital mirror equivalents by way of digital spaces, metrics which can translate into fully digital worlds as well.

For doing so we utilized the notion of presence, the sensation of being present in a virtual situation, as if actually there. This resulted in a framework that conceptualizes presence along social and spatial dimensions. Utilizing the framework, we then devised a set of metrics that capture increasingly rich forms of virtual visitation.

We acknowledge that many of the technologies at the heart of making such new forms of visitation are still under development and have not been widely adopted yet. Our framework is forward looking and will require future research and adaptation as technologies evolve.

Finally, we note that virtual social communities have evolved over time from simple text-based forms as in the case of Usenet, via virtual worlds such as Second Life, to rich game-based environments as in the case of Fortnite and others. At the same time the physical social events space is evolving, as museums, installations and others are rethinking their purpose and value using digital technology, and concerts are experimenting with virtual experiences.

\section{References}

[1] Novet, J. (2020) Zoom (ZM) earnings Q1 2021, $C N B C$.Available at: www.cnbc.com/2020/06/02/zoomzm-earnings-q1- 2021 (Accessed: 6 July 2020).

[2] Goslin, A. (2020) Fortnite's Travis Scott event drew over 27 million players, Polygon. Available at: https://www.polygon.com/fortnite/2020/4/24/2123 5017/fortnite-travis-scott-event-concert- astronomical- 12-3-million-concurrent-players- record (Accessed: 6 July 2020).

[3] Getz, D. and Page, S. J. (2014) 'Progress and prospects for event tourism research', Tourism Management. Elsevier Ltd, 52, pp. 593-631. doi: 10.1016/j.tourman.2015.03.007.

[4] Prifti, L., et al. A Competency Model for "Industrie 4.0" Employees. [ed.] J.M. Leimeister and W. Brenner. Proceedings der 13. Internationalen Tagung Wirtschaftsinformatik (WI 2017). St. Gallen : s.n., 2017, pp. 46-60.

[5] Qi, Q.,Tao, F. 2018, "Digital twin and big data towards smart manufacturing and Industry 4.0: 360 degree comparison", IEEE Access, Vol. 6 No. 1, pp. 35853593.)

[6] Luściński, S. (2018) 'Digital Twinning for Smart Industry'. doi: 10.4108/eai.6-11-2018.2279986.

[7] Nikolakis, N., Sipsas, K. and Makris, S. (2018) 'A cyber-physical context-aware system for coordinating human-robot collaboration', Procedia CIRP. Elsevier B.V., 72, pp. 27-32. doi: 10.1016/j.procir.2018.03.033.

[8] Schultze, U. (2010) 'Embodiment and presence in virtual worlds: A review', Journal of Information Technology. Palgrave Macmillan, 25(4), pp. 434- 449. doi: $10.1057 /$ jit.2010.25.

[9] Armstrong, J. K. (2016) Where does canned laughter come from and where did it go? - BBC Culture, $B B C$. Available at:

https://www.bbc.com/culture/article/20160926- wheredoes-canned-laughter-come-from-and- where-did-it-go (Accessed: 6 July 2020).

[10] Uhlemann, T. H. J., Lehmann, C. and Steinhilper, R. (2017) 'The Digital Twin: Realizing the CyberPhysical Production System for Industry 4.0', Procedia CIRP. The Author(s), 61, pp. 335-340. doi: 10.1016/j.procir.2016.11.152.

[11] Seymour, M., Riemer, K. and Kay, J. (2018) 'Actors, Avatars and Agents: Potentials and Implications of Natural Face Technology for the creation of Realistic Visual Presence', Journal of association for information systems.

[12] Riemer, K., Frößler, F. and Klein, S. (2007) 'Real Time Communication - Modes of Use in Distributed Teams', in 15th European Conference on Information Systems, St. Gallen (CH), 07- 09 June, pp. 286-297. doi: 10.1007/BF00357884.

[13] Altschuller, S. and Benbunan-Fich, R. (2013) 'The pursuit of trust in Ad hoc virtual teams: How much electronic portrayal is too much?', European Journal of Information Systems. doi: 10.1057/ejis.2012.39.

[14] Lee, K. M. (2004) 'Presence, Explicated', Communication Theory, 14(1), pp. 27-50. doi: $10.1093 / \mathrm{ct} / 14.1 .27$

[15] Lombard, M. and Ditton, T. (1997) 'At the Heart of it All. The Concept of Presence', Journal of ComputerMediated Communication, 3(September), p. 20. doi: $10.1093 / \mathrm{cid} / \mathrm{cir} 583$.

[16] Hartmann, T., Wirth, W., Schramm, H., Klimmt, C., Vorderer, P., Gysbers, A., Böcking, S., Ravaja, N., Laarni, J., Saari, T., Gouveia, F., \& Sacau, A. (2016). The Spatial Presence Experience Scale (SPES): A 
short self-report measure for diverse media settings. Journal of Media Psychology.

[17] Lombard, M., Ditton, T. B., \& Weinstein, L. (2009). Measuring presence: The Temple Presence Inventory. Proceedings of the International Society for Presence Research Annual Conference.

[18] Karis, D., Wildman, D. and Mané, A. (2016) 'Improving remote collaboration with video conferencing and video portals', HCI, 31(1), pp. 158. doi: 10.1080/07370024.2014.921506.

[19] Sallnäs, E.-L. (2005) 'Effects of Communication Mode on Social Presence, Virtual Presence, and Performance in Collaborative Virtual Environments', Presence: Teleoperators and Virtual Environments, 14(4), pp. 434-449. doi: $10.1162 / 105474605774785253$.

[20] Subramaniam, N., Nandhakumar, J. and Baptista John, J. (2013) 'Exploring social network interactions in enterprise systems: The role of virtual co-presence', Information Systems Journal, 23(6), pp. 475-499. doi: 10.1111/isj.12019.

[21] Slater, M. and Usoh, M. (1993) 'Representations Systems, Perceptual Position, and Presence in Immersive Virtual Environments', Presence: Teleoperators and Virtual Environments. doi: 10.1162/pres.1993.2.3.221.

[22] Tran, M. H., Yang, Y. and Raikundalia, G. K. (2005) 'Supporting awareness in instant messaging: an empirical study and mechanism design', in Proceedings of OZCHI'05, the CHISIG Annual Conference on Human-Computer Interaction.

[23] Dourish, P. and Bellotti, V. (1992) 'Awareness and coordination in shared workspaces', in Proceedings of the Conference on Computer-Supported Cooperative Work.

[24] Schmidt, K. (2002) "The Problem with "Awareness": Introductory Remarks on "Awareness in CSCW", Computer Supported Cooperative Work, 11, pp. 285298. 